\title{
Mixing, demixing, and structure formation in a binary dipolar Bose-Einstein condensate
}

\author{
Luis E. Young-S.* and S. K. Adhikari ${ }^{\dagger}$ \\ Instituto de Física Teórica, UNESP, Universidade Estadual Paulista, 01.140-070 São Paulo, São Paulo, Brazil
}

(Received 14 August 2012; revised manuscript received 21 September 2012; published 11 December 2012)

\begin{abstract}
We study the static properties of disk-shaped binary dipolar Bose-Einstein condensates of ${ }^{168} \mathrm{Er}-{ }^{164} \mathrm{Dy}$ and ${ }^{52} \mathrm{Cr}-$ ${ }^{164}$ Dy mixtures under the action of interspecies and intraspecies contact and dipolar interactions and demonstrate the effect of dipolar interaction using the mean-field approach. Throughout this study we use realistic values of interspecis and intraspecies dipolar interactions and the intraspecies scattering lengths and consider the interspecies scattering length as a parameter. The stability of the binary mixture is illustrated through phase plots involving a number of atoms of the species. The binary system always becomes unstable as the number of atoms increases beyond a certain limit. As the interspecies scattering length increases corresponding to more repulsion, an overlapping mixed state of the two species changes to a separated demixed configuration. During the transition from a mixed to a demixed configuration as the interspecies scattering length is increased for parameters near the stability line, the binary condensate shows special transient structures in density in the form of red-blood-cell-like biconcave and Saturn-ring-like shapes, which are direct manifestations of the dipolar interaction.
\end{abstract}

DOI: 10.1103/PhysRevA.86.063611

PACS number(s): $03.75 . \mathrm{Hh}, 03.75 . \mathrm{Mn}$

\section{INTRODUCTION}

After the experimental realization [1-5] of a dipolar BoseEinstein condensate (BEC) of ${ }^{52} \mathrm{Cr}$ atoms with magnetic moments, there has been renewed interest in the study of the static and dynamic properties of such a condensate in the pursuit of the novel and interesting properties and features emerging as a consequence of anisotropic long-range dipolar interaction. The atomic interaction in a dilute BEC of alkali-metal atoms is taken as an $s$-wave short-range (delta-function) potential. However, the anisotropic long-range dipolar interaction is nonlocal in nature acting in all partial waves. More recently, the BEC of ${ }^{164} \mathrm{Dy}[6,7]$ and ${ }^{168} \mathrm{Er}$ [8] atoms with larger dipole moments became available for experimental studies, and polar molecules with much larger (electric) dipole moments are being considered [9] for BEC experiments. Among the novel features of a BEC with anisotropic dipolar interaction, one can mention the peculiar shape and stability properties of a stationary state [10], a red-blood-cell-like biconcave shape in density due to radial and angular roton-like excitations [11]; anisotropic $d$-wave collapse [12]; the formation of an anisotropic soliton, vortex soliton [13], and vortex lattice [14]; anisotropic sound and shock wave propagation [15]; and anisotropic Landau critical velocity [16] among others. Distinct stable checkerboard, stripe, and star configurations in dipolar BECs have been identified in a two-dimensional (2D) optical lattice as a stable Mott insulator [17] as well as superfluid soliton [18] states. A new possibility of studying the universal properties of dipolar BECs for large scattering length has been suggested [19]. Many of these features have only been predicted theoretically, although some of them have already been experimentally confirmed. To enhance the effect of the anisotropic dipolar interaction in most of these theoretical studies, the contact interaction has been set equal to zero. In the ${ }^{52} \mathrm{Cr}$ atom, this

\footnotetext{
*1young@ift.unesp.br

†adhikari@ift.unesp.br; http://www.ift.unesp.br/users/adhikari
}

could be necessary as the strength of the repulsive contact interaction is much stronger than that of the dipolar interaction. However, the dipolar BEC of atoms such as ${ }^{164}$ Dy with much larger dipolar interaction can show the effects of an anisotropic nonlocal dipolar interaction without switching off the contact interaction.

Now with the available dipolar BECs of a different species of atoms, there is the possibility to investigate a binary mixture with two dipolar BECs of two types of dipolar interactions acting on each species (e.g., intraspecies and interspecies) superposed on intraspecis and interspecies contact interactions, thus creating a much richer platform to study the effect of dipolar interaction. Previously, there have been studies of nondipolar binary boson-boson [20,21], boson-fermion [22], and fermion-fermion [23], as well as dipolar-nondipolar binary boson-boson [24] mixtures. For a binary system without dipolar interactions it is possible to have either a mixed or demixed phase. In this paper, we consider binary dipolar BECs composed of ${ }^{164} \mathrm{Dy}$ and ${ }^{52} \mathrm{Cr}$ atoms as well as ${ }^{164} \mathrm{Dy}$ and ${ }^{168} \mathrm{Er}$ atoms. With all the dipole moments aligned along the $z$ axis, the net dipolar interaction is attractive in the cigar shape along the $z$ axis and repulsive in the disk shape confined in the $x-y$ plane, as parallel dipoles in a chain along the $z$ axis attract and those confined in the $x-y$ plane repeal each other. Consequently, the binary dipolar BEC with dipoles polarized along the $z$ axis is more stable in the disk shape confined in the $x-y$ plane compared to the cigar shape along the $z$ axis vulnerable to collapse. We shall consider only such a disk-shaped binary dipolar BEC in this study. Even such a disk-shaped binary dipolar BEC is found to be unstable due to the collapse instability for dipolar interaction above a critical value controlled by the number of atoms. This is consistent with a similar conclusion that a single-component dipolar BEC for any trap anisotropy collapses above a critical value of dipolar interaction [25,26].

We study the limits of the stability of binary dipolar disk-shaped BECs formed of ${ }^{168} \mathrm{Er}$ and ${ }^{164} \mathrm{Dy}$ atoms and of ${ }^{52} \mathrm{Cr}$ and ${ }^{164} \mathrm{Dy}$ atoms and present the results in terms of stability phase plots which should aid in the experimental 
preparation and study of binary dipolar BECs. Of all the atomic interactions, the interspecies and intraspecies dipolar interactions controlled by the known dipole moments of the two species will be considered known. The contact interactions governed by the approximately known intraspecies scattering lengths will also be taken as fixed parameters in this study. We then study the stability of the binary mixture by varying the number of atoms in each species and the yet unknown interspecies scattering length $a_{12}$. For small values of $a_{12}$, the binary dipolar BEC prefers a mixed configuration and with the augmentation of the interspecies repulsion the system moves into a demixed configuration. During this process of mixing-demixing, distinct biconcave red-blood-cell-like and Saturn-ring-like shapes are found in the densities of the two components near the stability line of the binary dipolar BEC for a certain value of interspecies scattering length. This configuration corresponds to saddle-point structures in the $2 \mathrm{D}$ densities of the two components in the $x-z$ plane. It was demonstrated [3] that the dipolar interaction corresponds to a saddle structure in two dimensions responsible for the saddle structure in density in the present study on binary dipolar BEC. The biconcave structure is a consequence of roton instability near the stability line due to dipolar interaction and was studied in detail in single-component dipolar BECs [11]. The Saturn-ring-like density distribution was never observed in a single-component dipolar BEC. To enhance the dipolar effect, in these previous studies the repulsive contact interaction was set to zero, whereas in the present study we set all the intraspecies and interspecies repulsive contact interactions to their large experimental values and yet obtain these special structures because of the larger values of dipole interactions appropriate for ${ }^{164} \mathrm{Dy}$ and ${ }^{168} \mathrm{Er}$ atoms.

In Sec. II we present the mean-field model for the binary dipolar BEC interacting via interspecies and intraspecies contact and dipolar interactions in an axially symmetric confinement. The details of the numerics together with an exposition of our numerical results are included in Sec. III. After the application of our model to a simplified binary dipolar BEC without any contact interaction, we present the results for the binary ${ }^{168} \mathrm{Er}-{ }^{164} \mathrm{Dy}$ and ${ }^{52} \mathrm{Cr}^{-164} \mathrm{Dy}$ mixtures. First, we obtain the stability phase plots of the binary dipolar BECs. Then we demonstrate mixing, demixing, and structure formation in densities in both cases. Close to the stability line, we find Saturn-ring-like and red-blood-cell-like density distributions in the components (only possible in the presence of dipolar interaction). Finally, in Sec. III we present a brief summary of our findings.

\section{MEAN-FIELD MODEL FOR THE BINARY DIPOLAR BEC}

We consider a general two-species dipolar BEC with interspecies and intraspecies dipolar and contact interactions with the mass, number, magnetic moment, and scattering length denoted by $m_{i}, N_{i}, \mu_{i}, a_{i}, i=1,2$, respectively. The angular frequencies for the axially symmetric trap along the $x, y$, and $z$ directions are taken as $\omega_{x}^{(i)}=\omega_{y}^{(i)}=\omega_{i}$ and $\omega_{z}=\lambda_{i} \omega_{i}$. The intraspecies and interspecies interactions for two atoms at positions $\mathbf{r}$ and $\mathbf{r}^{\prime}$ are taken as

$$
\begin{gathered}
V_{i}(\mathbf{R})=\frac{\mu_{0} \mu_{i}^{2}}{4 \pi} \frac{1-3 \cos ^{2} \theta}{|\mathbf{R}|^{3}}+\frac{4 \pi \hbar^{2} a_{i}}{m_{i}} \delta(\mathbf{R}), \\
V_{12}(\mathbf{R})=\frac{\mu_{0} \mu_{1} \mu_{2}}{4 \pi} \frac{1-3 \cos ^{2} \theta}{|\mathbf{R}|^{3}}+\frac{2 \pi \hbar^{2} a_{12}}{m_{R}} \delta(\mathbf{R}),
\end{gathered}
$$

where $\mathbf{R}=\mathbf{r}-\mathbf{r}^{\prime}, \mu_{0}$ is the permeability of free space, $\theta$ is the angle made by the vector $\mathbf{R}$ with the polarization in the $z$ direction, and $m_{R}=m_{1} m_{2} /\left(m_{1}+m_{2}\right)$ is the reduced mass of the two species of atoms. With these interactions, the coupled Gross-Pitaevskii (GP) equations for the binary dipolar BEC can be written as [1]

$$
\begin{aligned}
i \hbar \frac{\partial \phi_{1}(\mathbf{r}, t)}{\partial t}= & {\left[-\frac{\hbar^{2}}{2 m_{1}} \nabla^{2}+\frac{1}{2} m_{1} \omega_{1}^{2}\left(\rho^{2}+\lambda_{1}^{2} z^{2}\right)\right.} \\
& +N_{1} \frac{\mu_{0} \mu_{1}^{2}}{4 \pi} \int V_{d d}(\mathbf{R})\left|\phi_{1}\left(\mathbf{r}^{\prime}, t\right)\right|^{2} d \mathbf{r}^{\prime} \\
& +\frac{2 \pi \hbar^{2}}{m_{R}} a_{12} N_{2}\left|\phi_{2}(\mathbf{r}, t)\right|^{2}+\frac{4 \pi \hbar^{2}}{m_{1}} a_{1} N_{1}\left|\phi_{1}(\mathbf{r}, t)\right|^{2} \\
& \left.+N_{2} \frac{\mu_{0} \mu_{1} \mu_{2}}{4 \pi} \int V_{d d}(\mathbf{R})\left|\phi_{2}\left(\mathbf{r}^{\prime}, t\right)\right|^{2} d \mathbf{r}^{\prime}\right] \phi_{1}(\mathbf{r}, t),
\end{aligned}
$$

$$
\begin{aligned}
i \hbar \frac{\partial \phi_{2}(\mathbf{r}, t)}{\partial t}= & {\left[-\frac{\hbar^{2}}{2 m_{2}} \nabla^{2}+\frac{1}{2} m_{2} \omega_{2}^{2}\left(\rho^{2}+\lambda_{2}^{2} z^{2}\right)\right.} \\
& +N_{2} \frac{\mu_{0} \mu_{2}^{2}}{4 \pi} \int V_{d d}(\mathbf{R})\left|\phi_{2}\left(\mathbf{r}^{\prime}, t\right)\right|^{2} d \mathbf{r}^{\prime} \\
& +\frac{2 \pi \hbar^{2}}{m_{R}} a_{12} N_{1}\left|\phi_{1}(\mathbf{r}, t)\right|^{2}+\frac{4 \pi \hbar^{2}}{m_{2}} a_{2} N_{2}\left|\phi_{2}(\mathbf{r}, t)\right|^{2} \\
+ & \left.N_{1} \frac{\mu_{0} \mu_{1} \mu_{2}}{4 \pi} \int V_{d d}(\mathbf{R})\left|\phi_{1}\left(\mathbf{r}^{\prime}, t\right)\right|^{2} d \mathbf{r}^{\prime}\right] \phi_{2}(\mathbf{r}, t), \\
& V_{d d}(\mathbf{R})=\frac{1-3 \cos ^{2} \theta}{\mathbf{R}^{3}},
\end{aligned}
$$

with $\rho^{2}=x^{2}+y^{2}$.

To compare the dipolar and contact interactions, the intraspecies and interspecies dipolar interactions will be expressed in terms of the length scales $a_{d d}^{(i)}$ and $a_{d d}^{(12)}$, respectively, defined by

$$
\frac{\mu_{0} \mu_{i}^{2}}{4 \pi}=\frac{3 \hbar^{2}}{m_{i}} a_{d d}^{(i)}, \quad \frac{\mu_{0} \mu_{1} \mu_{2}}{4 \pi}=\frac{3 \hbar^{2}}{m_{1}} a_{d d}^{(12)} .
$$

For the intraspecies dipolar scale $a_{d d}^{(i)}$, the mass of the corresponding species $m_{i}$ has been used to define the scale. To define the interspecies dipolar scale $a_{d d}^{(12)}$, we have used the mass $m_{1}$ of the first species. We express the strengths of the dipolar interactions in Eqs. (3) and (4) by these length scales and transform these equations into the following dimensionless 
form:

$$
\begin{aligned}
i \frac{\partial \phi_{1}(\mathbf{r}, t)}{\partial t}= & {\left[-\frac{\nabla^{2}}{2}+\frac{1}{2}\left(\rho^{2}+\lambda_{1}^{2} z^{2}\right)+g_{1}\left|\phi_{1}\right|^{2}\right.} \\
& +g_{d d}^{(1)} \int V_{d d}(\mathbf{R})\left|\phi_{1}\left(\mathbf{r}^{\prime}, t\right)\right|^{2} d \mathbf{r}^{\prime}+g_{12}\left|\phi_{2}\right|^{2} \\
& \left.+g_{d d}^{(12)} \int V_{d d}(\mathbf{R})\left|\phi_{2}\left(\mathbf{r}^{\prime}, t\right)\right|^{2} d \mathbf{r}^{\prime}\right] \phi_{1}(\mathbf{r}, t), \\
i \frac{\partial \phi_{2}(\mathbf{r}, t)}{\partial t}= & {\left[-m_{12} \frac{\nabla^{2}}{2}+m_{w} \frac{1}{2}\left(\rho^{2}+\lambda_{2}^{2} z^{2}\right)+g_{2}\left|\phi_{2}\right|^{2}\right.} \\
& +g_{d d}^{(2)} \int V_{d d}(\mathbf{R})\left|\phi_{2}\left(\mathbf{r}^{\prime}, t\right)\right|^{2} d \mathbf{r}^{\prime}+g_{21}\left|\phi_{1}\right|^{2} \\
& \left.+g_{d d}^{(21)} \int V_{d d}(\mathbf{R})\left|\phi_{1}\left(\mathbf{r}^{\prime}, t\right)\right|^{2} d \mathbf{r}^{\prime}\right] \phi_{2}(\mathbf{r}, t),
\end{aligned}
$$

where $m_{12}=m_{1} / m_{2}, \quad m_{w}=\omega_{2}^{2} /\left(m_{12} \omega_{1}^{2}\right), \quad g_{1}=4 \pi a_{1} N_{1}$, $g_{d d}^{(1)}=3 N_{1} a_{d d}^{(1)}, \quad g_{2}=4 \pi a_{2} N_{2} m_{12}, \quad g_{12}=2 \pi m_{1} a_{12} N_{2} / m_{R}$, $g_{21}=2 \pi m_{1} a_{12} N_{1} / m_{R}, g_{d d}^{(2)}=3 N_{2} a_{d d}^{(2)} m_{12}, g_{d d}^{(12)}=3 N_{2} a_{d d}^{(12)}$, and $g_{d d}^{(21)}=3 N_{1} a_{d d}^{(12)}$. In Eqs. (7) and (8), length is expressed in units of oscillator length for the first species $l_{0}=\sqrt{\hbar / m_{1} \omega_{1}}$, energy in units of oscillator energy $\hbar \omega_{1}$, density $\left|\phi_{i}\right|^{2}$ in units of $l_{0}^{-3}$, and time in units of $t_{0}=\omega_{1}^{-1}$.

\section{NUMERICAL RESULTS}

For the binary dipolar BEC we solve Eqs. (7) and (8) numerically after discretization [27]. The divergence of the dipolar term at short distances has been handled by treating this term in momentum (k) space. The dipolar integral in (Fourier) momentum space is tackled by the following convolution integral $[1,28]$ :

$$
\int d \mathbf{r}^{\prime} V_{d d}(\mathbf{R}) n\left(\mathbf{r}^{\prime}\right)=\int \frac{d \mathbf{k}}{(2 \pi)^{3}} e^{-i \mathbf{k} \cdot \mathbf{r}} V_{d d}(\mathbf{k}) n(\mathbf{k}),
$$

with $n(\mathbf{r})=|\phi(\mathbf{r})|^{2}$. The Fourier transformation (FT) is defined by

$$
\begin{gathered}
A(\mathbf{k})=\int d \mathbf{r} B(\mathbf{r}) e^{i \mathbf{k} \cdot \mathbf{r}}, \\
B(\mathbf{r})=\frac{1}{(2 \pi)^{3}} \int d \mathbf{k} A(\mathbf{k}) e^{-i \mathbf{k} \cdot \mathbf{r}} .
\end{gathered}
$$

The FT $V_{d d}(\mathbf{k})$ of the dipolar potential is known analytically $[1,28]$

$$
V_{d d}(\mathbf{k})=\frac{4 \pi}{3}\left(\frac{3 k_{z}^{2}}{\mathbf{k}^{2}}-1\right) .
$$

The FT $n(\mathbf{k})$ of density is calculated numerically by a fast FT (FFT) routine. The inverse FT is also evaluated numerically by the FFT routine. The whole procedure is performed in a threedimensional (3D) Cartesian coordinate system irrespective of the underlying trap symmetry.

\section{A. Same species of atoms in isotropic trap}

To test the model and the numerical routine, we consider a simple binary dipolar BEC of the same species of atoms without contact interaction in a spherically symmetric trap. However, the two components are considered to be oppositely polarized along the $z$ and $-z$ directions, respectively. In this model, first considered by Góral and Santos [28], $m_{i}=m, \omega_{i}=\omega, a_{d d}^{(i)}=-a_{d d}^{(12)}=a_{d d}, a_{i}=$ $a_{12}=0, m_{12}=m_{\omega}=1, \lambda_{i}=1$. Consequently, in Eqs. (7) and (8) $g_{i}=g_{12}=0, g_{d d}^{(i)}=3 N_{i} a_{d d}, g_{d d}^{(12)}=-3 N_{2} a_{d d}$, and $g_{d d}^{(21)}=-3 N_{1} a_{d d}$. Using $\eta=N_{1} / N, \zeta=3 a_{d d} N, N=N_{1}+$ $\mathrm{N}_{2}$ we can write Eqs. (7) and (8) as in Ref. [28]

$$
\begin{aligned}
& i \frac{\partial \phi_{1}}{\partial t}=\left[-\frac{\nabla^{2}}{2}+\frac{r^{2}}{2}+\zeta\left\{\eta U_{1}(\mathbf{r})-(1-\eta) U_{2}(\mathbf{r})\right\}\right] \phi_{1}, \\
& i \frac{\partial \phi_{2}}{\partial t}=\left[-\frac{\nabla^{2}}{2}+\frac{r^{2}}{2}+\zeta\left\{(1-\eta) U_{2}(\mathbf{r})-\eta U_{1}(\mathbf{r})\right\}\right] \phi_{2},
\end{aligned}
$$

$$
U_{i}(\mathbf{r})=\int V_{d d}(\mathbf{R})\left|\phi_{i}\left(\mathbf{r}^{\prime}\right)\right|^{2} d \mathbf{r}^{\prime}, \quad i=1,2 .
$$

For a fixed fraction of the atoms of the first component $\eta$, the system is stable up to a critical value of net dipolar interaction nonlinearity $\zeta$ (e.g., for $\zeta<\zeta_{\text {cr }}$ ) beyond which the system becomes unstable. The stability of the binary system can be expressed in terms of a $\zeta-\eta$ phase diagram. This stability phase diagram for this binary dipolar condensate of the same species of atoms, but of oppositely polarized dipolar moments, in a spherically symmetric trap is shown in Fig. 1 in agreement with Góral and Santos [28]. Independent of the composition of the system governed by the parameter $\eta$, the system is unstable beyond a critical value of the total number of atoms $N$.

\section{B. Different species of atoms in disk trap}

Binary ${ }^{168} \mathrm{Er}-{ }^{164}$ Dy mixture. We consider two different binary mixtures of dipolar BECs. First, we consider the ${ }^{168} \mathrm{Er}-$ ${ }^{164} \mathrm{Dy}$ mixture. In this case we take BEC number 1 as ${ }^{168} \mathrm{Er}$ and BEC number 2 as ${ }^{164}$ Dy with parameters $\mu_{1}=7 \mu_{B}, \mu_{2}=$ $10 \mu_{B}, \quad a_{d d}^{(1)}=66 a_{0}, a_{d d}^{(12)}=94 a_{0}, a_{d d}^{(2)}=131 a_{0}$ with $a_{0}$ the Bohr radius and $\mu_{B}$ the Bohr magneton. Without accurate experimental estimates $[7,8]$ of the intraspecies scattering lengths, we use $a_{i}=110 a_{0}$. The angular frequencies of the axial trap for ${ }^{164}$ Dy are taken as $\omega_{2}=2 \pi \times 243 \mathrm{~Hz}, \lambda_{2}=$ $\sqrt{10} \approx 3.1623$, compared with the experimental frequencies

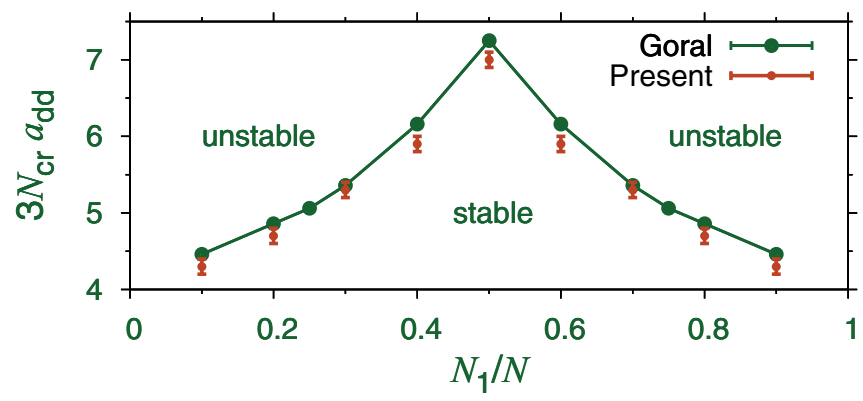

FIG. 1. (Color online) The maximum allowed value of net intraspecies dipolar nonlinearity $\zeta_{\mathrm{cr}}\left(\equiv 3 N_{\mathrm{cr}} a_{d d}\right)$ versus fraction of atoms of the first component $\eta\left(\equiv N_{1} / N\right)$ for a binary dipolar BEC of oppositely polarized dipolar gases in a spherically symmetric trap compared with the findings of Góral and Santos [28]. 
[7] $\left\{f_{x}, f_{y}, f_{z}\right\}=\{205,195,760\} \mathrm{Hz}$ with trap aspect ratio $\lambda=3.8$. We take $m_{\omega}=\left(m_{2} \omega_{2}^{2}\right) /\left(m_{1} \omega_{1}^{2}\right)=1$, corresponding to $\omega_{1}=2 \pi \times 240 \mathrm{~Hz}, \lambda_{1}=\sqrt{10}$. The unit of length in this study is $l_{0}=\sqrt{\hbar /\left(m_{1} \omega_{1}\right)} \approx 0.5 \mu \mathrm{m}$ and the unit of time $t_{0}=\omega_{1}^{-1} \approx 0.663 \mathrm{~ms}$.

Binary ${ }^{52} \mathrm{Cr}-{ }^{164} \mathrm{Dy}$ mixture. In this case, taking the condensate number 1 as ${ }^{52} \mathrm{Cr}$ and the one number 2 as ${ }^{164} \mathrm{Dy}$, the parameters are $\mu_{1}=6 \mu_{B}, \mu_{2}=10 \mu_{B}, a_{d d}^{(1)}=15 a_{0}, a_{d d}^{(2)}=$ $131 a_{0}, a_{d d}^{(12)}=25 a_{0}$. We take $a_{i}=110 a_{0}$, close to their experimental estimates $[4,5,7]$. The angular frequencies for the axial trap for ${ }^{52} \mathrm{Cr}$ are taken as $\omega_{1}=2 \pi \times 195 \mathrm{~Hz}, \lambda_{1}=$ $\sqrt{10} \approx 3.1623$, compared with the experimental frequencies [4] $\left\{f_{x}, f_{y}, f_{z}\right\}=\left\{150,150, \lambda f_{x}\right\} \mathrm{Hz}$ with trap aspect ratio $\lambda<10$. We take $m_{\omega}=\left(m_{2} \omega_{2}^{2}\right) /\left(m_{1} \omega_{1}^{2}\right)=1$, corresponding to $\omega_{2}=2 \pi \times 110 \mathrm{~Hz}, \lambda_{2}=\sqrt{10}$. The unit of length in this calculation is $l_{0}=\sqrt{\hbar /\left(m_{1} \omega_{1}\right)} \approx 1 \mu \mathrm{m}$ and the unit of time $t_{0}=\omega_{1}^{-1} \approx 0.816 \mathrm{~ms}$.

\section{Stability phase plot}

By solving the coupled set of GP equations (7) and (8) for the binary mixture of dipolar BECs with the above parameters we find that the system becomes unstable above a certain number of atoms with the total dipolar interaction beyond a limiting value in agreement with a similar conclusion [25] in a single-component dipolar BEC. To perform a systematic study of the stability of the binary dipolar BEC, we solve Eqs. (7) and (8) with the above parameters, fixing the number of atoms in the first species $\left({ }^{52} \mathrm{Cr}\right.$ or $\left.{ }^{168} \mathrm{Er}\right)$ and searching for the critical number of atoms $\left(N_{\mathrm{cr}}\right)$ of the second species ${ }^{164}$ Dy beyond which the system becomes unstable for different values of the interspecies scattering length $a_{12}$. If the binary mixture has a smaller number of atoms of the first species $\left({ }^{52} \mathrm{Cr}\right.$ or $\left.{ }^{168} \mathrm{Er}\right)$, it can accommodate a larger number of atoms of the second species $\left({ }^{164} \mathrm{Dy}\right)$ while the limiting value of the total dipolar interaction is reached maintaining all other parameters fixed.

We present the results of our study in stability phase plots in Figs. 2. In this figure we show the critical number of ${ }^{164}$ Dy atoms $N_{\text {cr }}\left(\right.$ Dy) versus $a_{12}$ for 1000 and $10000{ }^{52} \mathrm{Cr}$ or ${ }^{168} \mathrm{Er}$ atoms in binary ${ }^{52} \mathrm{Cr}-{ }^{164} \mathrm{Dy}$ and ${ }^{168} \mathrm{Er}-{ }^{164} \mathrm{Dy}$ mixtures, respectively. The system is stable below the lines of Figs. 2 and unstable above. The stability phase plots of Figs. 2 could be relevant for planning future experiments on binary dipolar BEC. The system may have distinct structures in density in the shaded regions near the stability line in this figure. This region is similar to the darker region in Fig. 1 of Ref. [25], where the biconcave shape in density of a single-component dipolar BEC appeared due to roton instability. The biconcave shape in density in a disk-shaped dipolar BEC emerges because of dipolar repulsion in the plane of the disk. Due to this repulsion the atoms come to the peripheral region of the disk and a region of low density appears in the center. A similar low-density central region may appear in a rotating BEC due to centrifugal repulsion, as has been found in a binary nondipolar BEC in a rotating trap [21]. We shall see below that biconcave and Saturn-ring-like shapes in density of the components of the binary dipolar BEC may appear in the shaded region in Figs. 2. The system can accommodate a larger number of atoms for large positive values of $a_{12}$ responsible for large
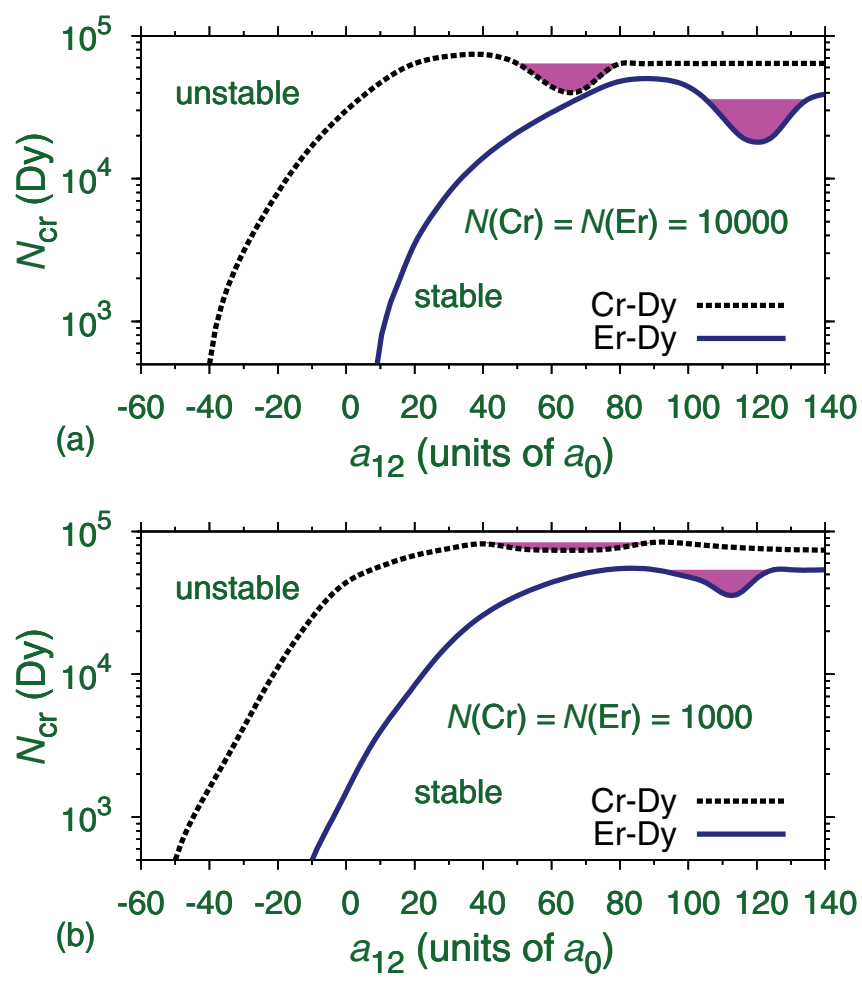

FIG. 2. (Color online) Stability phase plot showing the critical number $N_{\text {cr }}$ (Dy) of ${ }^{164}$ Dy atoms in the ${ }^{52} \mathrm{Cr}-{ }^{164} \mathrm{Dy}$ and ${ }^{168} \mathrm{Er}-{ }^{164} \mathrm{Dy}$ binary mixtures versus interspecies scattering length $a_{12}$ for (a) 10000 and (b) $1000{ }^{52} \mathrm{Cr}$ or ${ }^{168} \mathrm{Er}$ atoms, respectively. The system is stable below the respective lines. The shaded dark areas illustrate the domains, where red-blood-cell-like biconcave and Saturn-ring-like density profiles appear.

contact repulsion which stabilize the binary dipolar BEC. As $a_{12}$ reduces, the contact repulsion reduces and the system becomes more vulnerable to collapse for a larger number of atoms due to dipolar interaction and hence can accommodate only a small number of ${ }^{164}$ Dy atoms, as can be seen in Figs. 2.

\section{Mixing, demixing, and structure formation}

Next we study in detail the density of the two species of atoms in the binary dipolar BEC to look for mixing (overlapping phases of two components), demixing (separated phases), and the distinct structure formation in the shaded regions of the phase plots shown in Figs. 2. For small $a_{12}$ the interatomic contact repulsion between the two species is small and one has a mixed configuration. As $a_{12}$ is increased the interspecies contact repulsion increases and for a large-enough value of $a_{12}$ one can have demixing of the two species. In the demixed configuration one of the species occupies the central region and the other the external region. To illustrate this phenomenon, we plot in Fig. 3 the reduced 2D density in the radial direction $|\Phi(x, y=0)|^{2}=\int d z|\phi(x, y=0, z)|^{2}$ versus $x$ under different situations in the case of the binary ${ }^{168} \mathrm{Er}-{ }^{164} \mathrm{Dy}$ mixture. The mixing-demixing is illustrated in Figs. 3(a), 3(b), and 3(c) for $10000{ }^{168} \mathrm{Er}$ and $20000{ }^{164} \mathrm{Dy}$ atoms, respectively, for $a_{12}=110 a_{0}, 125 a_{0}$, and $130 a_{0}$. In Fig. 3(a) we have a mixed (overlapping) configuration and with the increase of interspecies contact repulsion, in Fig. 3(c) we 

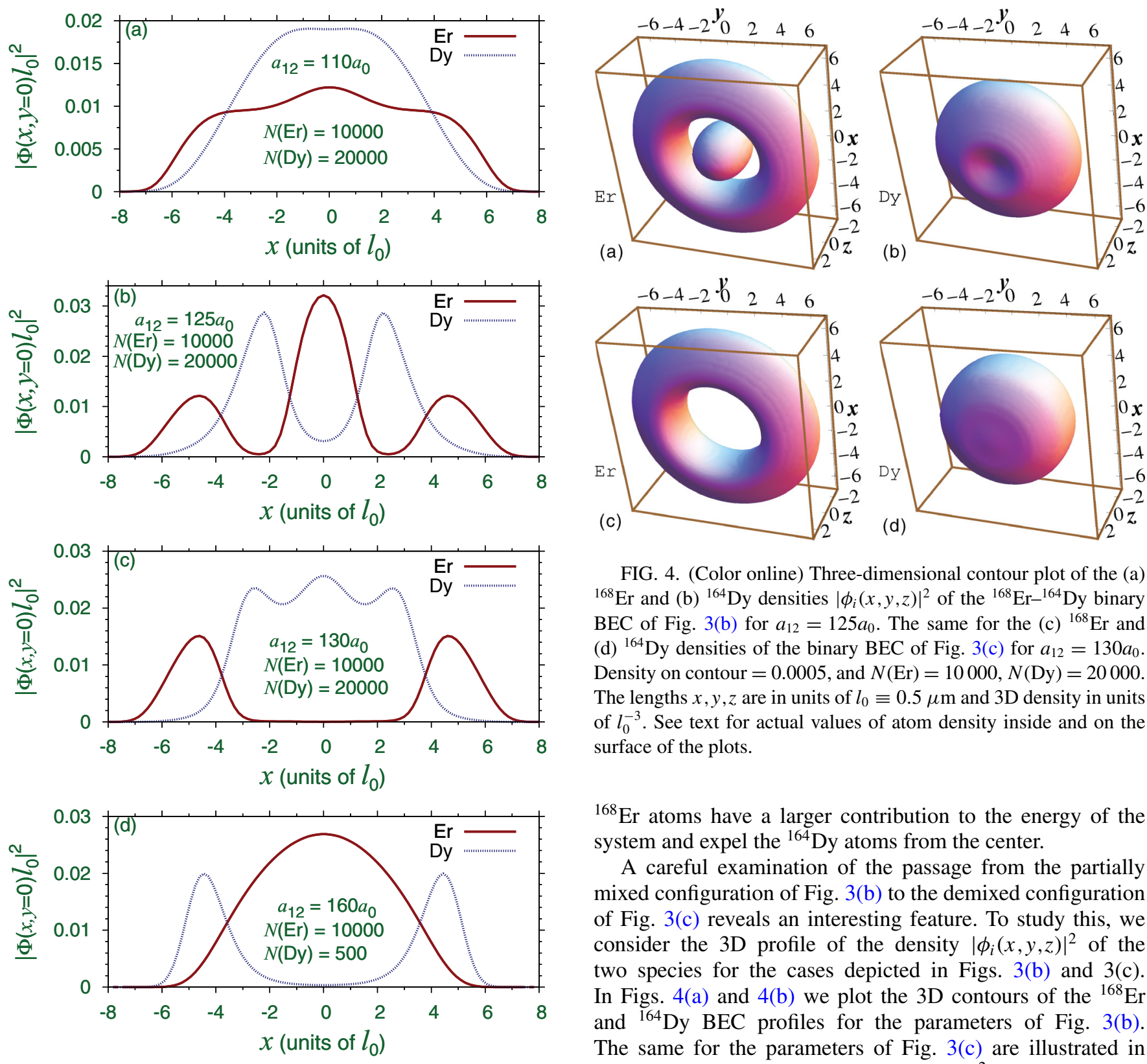

FIG. 3. (Color online) Two-dimensional radial density along the $x$ axis $|\Phi(x, y=0)|^{2} \equiv \int d z|\phi(x, y=0, z)|^{2}$ of the binary ${ }^{168} \mathrm{Er}-{ }^{164} \mathrm{Dy}$ $\mathrm{BEC}$, for $N(\mathrm{Er})=10000, N(\mathrm{Dy})=20000$, (a) $a_{12}=110 a_{0}$; (b) $a_{12}=125 a_{0}$; (c) $a_{12}=130 a_{0}$; and for $N(\mathrm{Er})=10000, N(\mathrm{Dy})=$ 500 , (d) $a_{12}=160 a_{0}$. For the trap parameters of this study the length scale $l_{0}=0.5 \mu \mathrm{m}$.

have a demixed (separated) configuration of the two species. In Fig. 3(b), for $a_{12}=125 a_{0}$ we have a partially demixed configuration. In the demixed configuration in Fig. 3(c), 20000 ${ }^{164}$ Dy atoms have a larger contribution to energy of the system and the ${ }^{164} \mathrm{Dy}$ atoms expel the ${ }^{168} \mathrm{Er}$ atoms from the central region due to interspecies repulsion. The opposite panorama is also possible for a larger number of ${ }^{168} \mathrm{Er}$ atoms, while the ${ }^{168} \mathrm{Er}$ atoms can expel the ${ }^{164} \mathrm{Dy}$ atoms from the central region. Such a situation is illustrated in Fig. 3(d) with $10000{ }^{168} \mathrm{Er}$ atoms and $500{ }^{164} \mathrm{Dy}$ atoms for $a_{12}=160 a_{0}$. In this case the

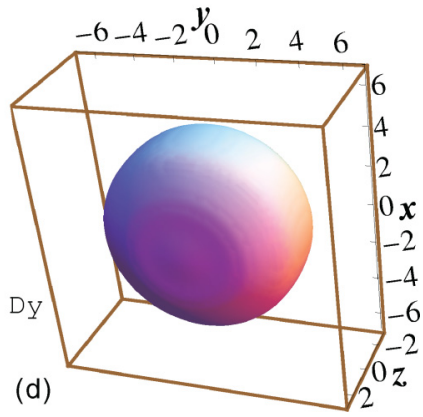

(d)

FIG. 4. (Color online) Three-dimensional contour plot of the (a) ${ }^{168} \mathrm{Er}$ and (b) ${ }^{164} \mathrm{Dy}$ densities $\left|\phi_{i}(x, y, z)\right|^{2}$ of the ${ }^{168} \mathrm{Er}-{ }^{164} \mathrm{Dy}$ binary BEC of Fig. 3(b) for $a_{12}=125 a_{0}$. The same for the (c) ${ }^{168} \mathrm{Er}$ and (d) ${ }^{164}$ Dy densities of the binary BEC of Fig. 3(c) for $a_{12}=130 a_{0}$. Density on contour $=0.0005$, and $N(\mathrm{Er})=10000, N(\mathrm{Dy})=20000$. The lengths $x, y, z$ are in units of $l_{0} \equiv 0.5 \mu \mathrm{m}$ and $3 \mathrm{D}$ density in units of $l_{0}^{-3}$. See text for actual values of atom density inside and on the surface of the plots.

${ }^{168} \mathrm{Er}$ atoms have a larger contribution to the energy of the system and expel the ${ }^{164}$ Dy atoms from the center.

A careful examination of the passage from the partially mixed configuration of Fig. 3(b) to the demixed configuration of Fig. 3(c) reveals an interesting feature. To study this, we consider the $3 \mathrm{D}$ profile of the density $\left|\phi_{i}(x, y, z)\right|^{2}$ of the two species for the cases depicted in Figs. 3(b) and 3(c). In Figs. 4(a) and 4(b) we plot the 3D contours of the ${ }^{168} \mathrm{Er}$ and ${ }^{164}$ Dy BEC profiles for the parameters of Fig. 3(b). The same for the parameters of Fig. 3(c) are illustrated in Figs. 4(c) and 4(d). The density $|\phi(x, y, z)|^{2}$ on the surface of these plots is $0.0005 l_{0}^{-3}$. With $l_{0}=0.5 \mu \mathrm{m}$, and $N(\mathrm{Er})=$ 10000 , and $N(\mathrm{Dy})=20000$ this leads to atom densities on contour of $40 \mu \mathrm{m}^{-3}$ for ${ }^{168} \mathrm{Er}$ and of $80 \mu \mathrm{m}^{-3}$ for ${ }^{164} \mathrm{Dy}$. The maxima of the atom density in the interior of the BECs of Figs. 4(a) through 4(d) are, respectively, 800, 1160, 460, and $1000 \mu \mathrm{m}^{-3}$. In Figs. 4(a) and 4(b) the ${ }^{168} \mathrm{Er}$ and ${ }^{164}$ Dy BECs have Saturn-ring-like and red-blood-cell-like biconcave profiles, respectively. The biconcave profile is a manifestation of the dipolar interaction: The net dipolar repulsion in disk-shaped ${ }^{164}$ Dy drives the dysprosium atoms to peripheral region thus creating the biconcave shape as in the single-component dipolar BEC. In the single-component case the biconcave profile near the stability limit has been related to roton instability [25]. The Saturn-ring-like ${ }^{168} \mathrm{Er}$ profile in Fig. 4(a) is a manifestation of the interspecies dipolar interaction: The biconcave density distribution of the ${ }^{164} \mathrm{Dy}$ species with a density minimum at center, due to interspecies repulsion, expels a part of the ${ }^{168} \mathrm{Er} \mathrm{BEC}$ to the central region of 
lower density and another part to the peripheral region, also of lower density, thus creating the Saturn-ring-like profile. Such a profile is not possible in a single-component dipolar BEC, or in a binary BEC without dipolar interaction. With further increase of interspecies contact repulsion, for $a_{12}=130 a_{0}$, the contact repulsions dominate over the dipolar interactions and hence plays a major role thus creating a simpler situation of demixing shown in Fig. 3(c) and Figs. 4(c) and 4(d), where the net interspecies repulsion is so strong that all ${ }^{168} \mathrm{Er}$ atoms are driven to the peripheral region in the form of a ring with the dominant ${ }^{164}$ Dy atoms occupying the central region. The ring-like structure in Fig. 4(a) as opposed to a shelllike demixed configuration is a consequence of the dipolar interaction: A pure interspecies contact repulsion would have led to a hollow shell-like configuration for ${ }^{168} \mathrm{Er}$ surrounding a compact disk-shaped ${ }^{164}$ Dy core. The dipolar interaction is attractive in the $z$ direction and this transforms the shell-like configuration into a ring. Such a transition from a shell-like configuration to a ring with the increase of dipolar interaction has been demonstrated in a single-component dipolar BEC in a shell-like trap [29].

From Fig. 3 we find that for a binary dipolar BEC composed of $10000{ }^{168} \mathrm{Er}$ atoms and $20000{ }^{164} \mathrm{Dy}$ atoms the transition from a mixed to a demixed configuration happens around $a_{12}=120 a_{0}$. We verified that a similar transition also takes place in a binary dipolar BEC for $10000{ }^{168} \mathrm{Er}$ atoms and a reduced number of ${ }^{164}$ Dy atoms also for $a_{12} \approx 120 a_{0}$. Nevertheless, the biconcave structure in the density of ${ }^{164} \mathrm{Dy}$ is obtained for a small shaded region near the stability line in Fig. 2(a) with a large number of ${ }^{164}$ Dy atoms. As in the single component case [25], the biconcave and Saturn-ring-like shapes in density in the binary dipolar BEC in the shaded region in Fig. 2(a) is usually transient as instability appears at large times after crossing the stability line. The present structures were obtained by using imaginary-time propagation of the coupled mean-field equations with Gaussian profiles for initial densities. We started with the Gaussian profiles of the linear oscillator states and increased the dipolar and nondipolar nonlinearities in small steps during numerical simulation until the final nonlinearities are reached. In this fashion the structure in density in the shaded regions of phase plots in Figs. 2 are obtained.

To gain further insight into the density distributions in Figs. 4(a) to 4(d) we plot in Figs. 5(a) to 5(d) contour plots of asymmetric 2D density $|\Phi(x, z)|^{2}=\int d y|\phi(x, y, z)|^{2}$ of the binary ${ }^{168} \mathrm{Er}-{ }^{164} \mathrm{Dy}$ mixture for parameters corresponding to Figs. 4(a) through 4(d), respectively. The double- and single-saddle structures in density in the $x-z$ plane in Figs. 5(a) and 5(b) are manifestations of the dipolar interaction. In these plots a minimum in density along the $x$ direction coincides with a maximum in density in the $z$ direction thus creating a saddle point, which appears as a clear manifestation of the saddle-shaped dipolar interaction [3]. Such a density distribution is not possible in the absence of dipolar interaction and to the best of our knowledge has not been demonstrated even in the single-component dipolar BEC. In Fig. 5(a), for $a_{12}=125 a_{0}$, we have two saddle points on the $x$ axis in the density of ${ }^{168} \mathrm{Er}$ and one saddle point in the density of ${ }^{164} \mathrm{Dy}$. Similarly, for $a_{12}=130 a_{0}$, we have a single saddle point only in the density of ${ }^{168} \mathrm{Er}$ and none in ${ }^{164} \mathrm{Dy}$. These saddle

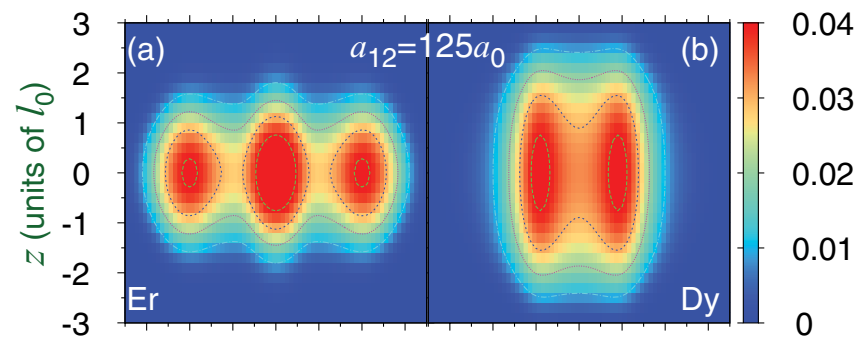

$\begin{array}{llllllllllllll}-6 & -4 & -2 & 0 & 2 & 4 & 6 & -6 & -4 & -2 & 0 & 2 & 4 & 6\end{array}$ $x$ (units of $\left.l_{0}\right) \quad x$ (units of $l_{0}$ )

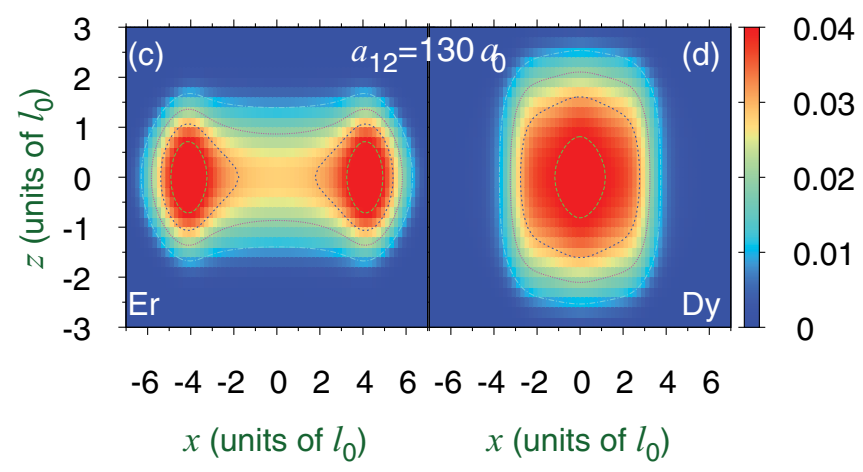

FIG. 5. (Color online) Contour plot of 2D density $|\Phi(x, z)|^{2} \equiv$ $\int d y|\phi(x, y, z)|^{2}$ of the binary ${ }^{168} \mathrm{Er}-{ }^{164} \mathrm{Dy} \mathrm{BEC}$ for $N(\mathrm{Er})=10000$, $N($ Dy $)=20000$ with $a_{12}=125 a_{0}$ for (a) ${ }^{168} \mathrm{Er}$, (b) ${ }^{164} \mathrm{Dy}$, and with $a_{12}=130 a_{0}$ for (c) ${ }^{168} \mathrm{Er}$ and (d) ${ }^{164} \mathrm{Dy}$. The lengths are expressed in units of $l_{0}(=0.5 \mu \mathrm{m})$ and the $2 \mathrm{D}$ density in units of $l_{0}^{-2}$.

points have curvatures consistent with saddle-shaped dipolar interaction [3] with negative curvature along the direction of magnetization ( $z$ axis) and positive curvature orthogonal to it $(x$ axis).

In Fig. 6 we plot the chemical potential $\mu$ and root mean square (rms) sizes $\langle z\rangle$, and $\langle\rho\rangle$ versus interspecies scattering length $a_{12}$, for (a) $N(\mathrm{Er})=N(\mathrm{Dy})=10000$; (b) $N(\mathrm{Er})=$ $10000, N(\mathrm{Dy})=1000$; (c) $N(\mathrm{Er})=1000, N(\mathrm{Dy})=10000$. It is found that the chemical potential and rms sizes are slowly varying functions of the interspecies scattering length. As expected, the chemical potential of the first species increases more rapidly with $a_{12}$ when there are more atoms in the second species and vice versa.

With this study of the binary ${ }^{168} \mathrm{Er}-{ }^{164} \mathrm{Dy} \mathrm{BEC}$, we also considered a detailed investigation of the binary ${ }^{52} \mathrm{Cr}-\mathrm{Dy}$ BEC. Similar mixing, demixing, and structure formation are also found in this second case, although the quantitative results and estimates are different. We only report here the studies on the interesting structure formation in the binary ${ }^{52} \mathrm{Cr}-{ }^{164} \mathrm{Dy} \mathrm{BEC}$. Indeed we find that one can have the condensate with biconcave shape in this case. In Figs. 7(a) and 7(b) we plot the linear density in the $x$ - $y$ plane along the $x$ direction $|\Phi(x, y=0)|^{2}$, as in Fig. 3, for a binary mixture of $10000{ }^{52} \mathrm{Cr}$ atoms and 60000 ${ }^{164} \mathrm{Dy}$ atoms for $a_{12}=70 a_{0}$ and $80 a_{0}$, respectively. Figure 7(a) illustrates a case similar to Fig. 3(b) denoting a transition from a mixed state to a demixed state. For $a_{12}<60 a_{0}$, we have a fully mixed configuration of the two condensates as in Fig. 3(a). For $a_{12}=70 a_{0}$ a biconcave shape has appeared in the density of ${ }^{52} \mathrm{Cr}$. For $a_{12}>75 a_{0}$, a complete demixed 

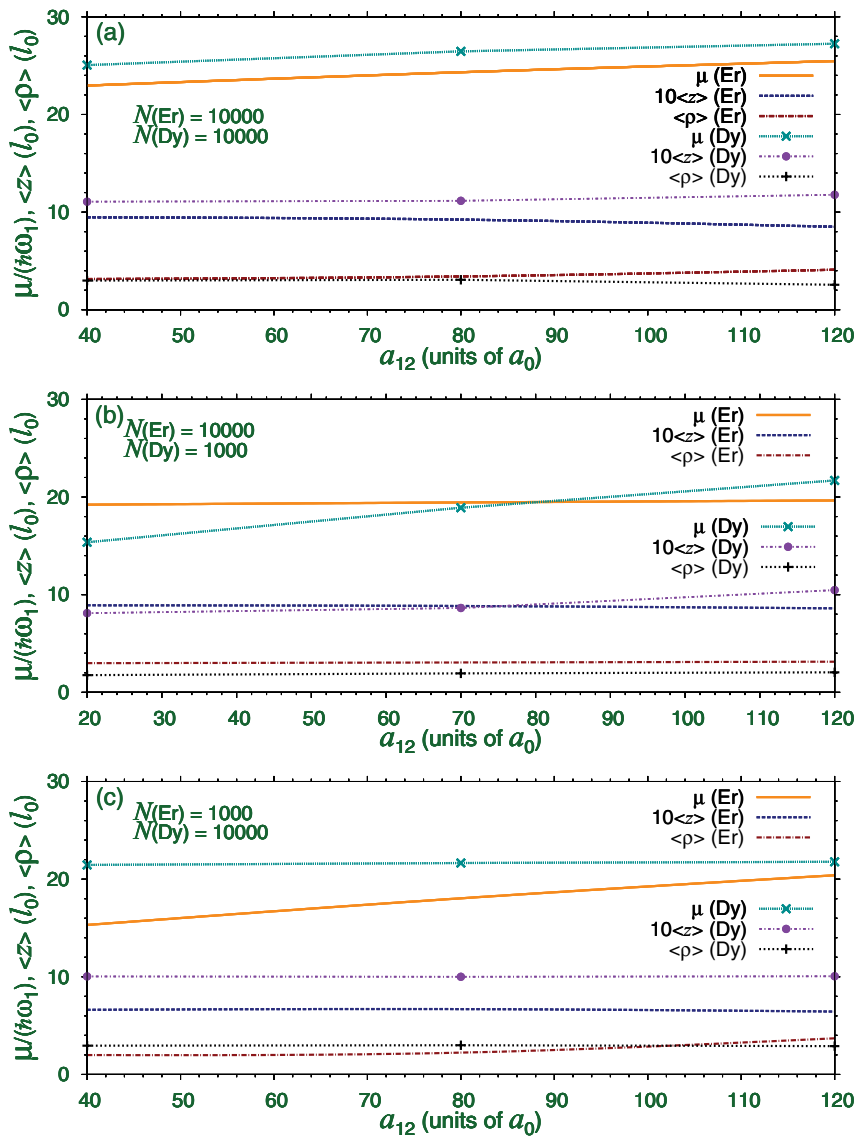

FIG. 6. (Color online) Chemical potential $\mu$ and rms sizes $\langle z\rangle,\langle\rho\rangle$ versus interspecies scattering length for a binary ${ }^{168} \mathrm{Er}-{ }^{164} \mathrm{Dy} \mathrm{BEC}$ for (a) $N(\mathrm{Er})=N(\mathrm{Dy})=10000$; (b) $N(\mathrm{Er})=10000, N(\mathrm{Dy})=1000$; (c) $N(\mathrm{Er})=1000, N(\mathrm{Dy})=10000$. The unit of length is $l_{0}=0.5 \mu \mathrm{m}$.

configuration appears as illustrated in Fig. 7(b) for $a=80 a_{0}$, where the ${ }^{52} \mathrm{Cr}$ atoms are expelled from the central region occupied by only ${ }^{164}$ Dy atoms. In Figs. 7(c) and 7(d) we show the 3D contour of the density $|\phi(x, y, z)|^{2}$ of ${ }^{52} \mathrm{Cr}$ and ${ }^{164} \mathrm{Dy}$ BECs corresponding to the parameters of Fig. 7(a) with the density $0.0005 l_{0}^{-3}$ on contour. This corresponds to the number densities on the contour of 5 and $30 \mu \mathrm{m}^{-3}$ in ${ }^{52} \mathrm{Cr}$ and ${ }^{164} \mathrm{Dy}$, respectively, for $l_{0}=1 \mu \mathrm{m}$. The ${ }^{52} \mathrm{Cr} \mathrm{BEC}$ shows a pronounced biconcave shape which is a direct manifestation of the dipolar interaction. However, we could not find a biconcave shape in the density of the ${ }^{164}$ Dy BEC, as in the case of a binary dipolar ${ }^{168} \mathrm{Er}-{ }^{164} \mathrm{Dy}$ mixture, possibly because the interspecies dipole interaction in the present system is much smaller in strength compared to the same in the binary ${ }^{168} \mathrm{Er}-{ }^{164} \mathrm{Dy}$ system so as to create the biconcave shape in density in the presence of dominating large contact repulsions acting on ${ }^{164} \mathrm{Dy}$. The much larger dipole interactions in ${ }^{164} \mathrm{Dy}$ might be responsible for the biconcave shape in density in Fig. 4(b). Even in the singlecomponent case, the biconcave profile in density appears for certain values of the trap aspect ratio [25], and it is also possible that for other values of parameters the biconcave profile might appear in the density of ${ }^{164} \mathrm{Dy}$ in the present binary ${ }^{52} \mathrm{Cr}-{ }^{164} \mathrm{Dy}$ mixture. However, the dipolar interaction leaves its signature in a different fashion as can be seen in Figs. 7(e) and 7(f), where we plot the same densities of Figs. 7(c) and 7(d), but now
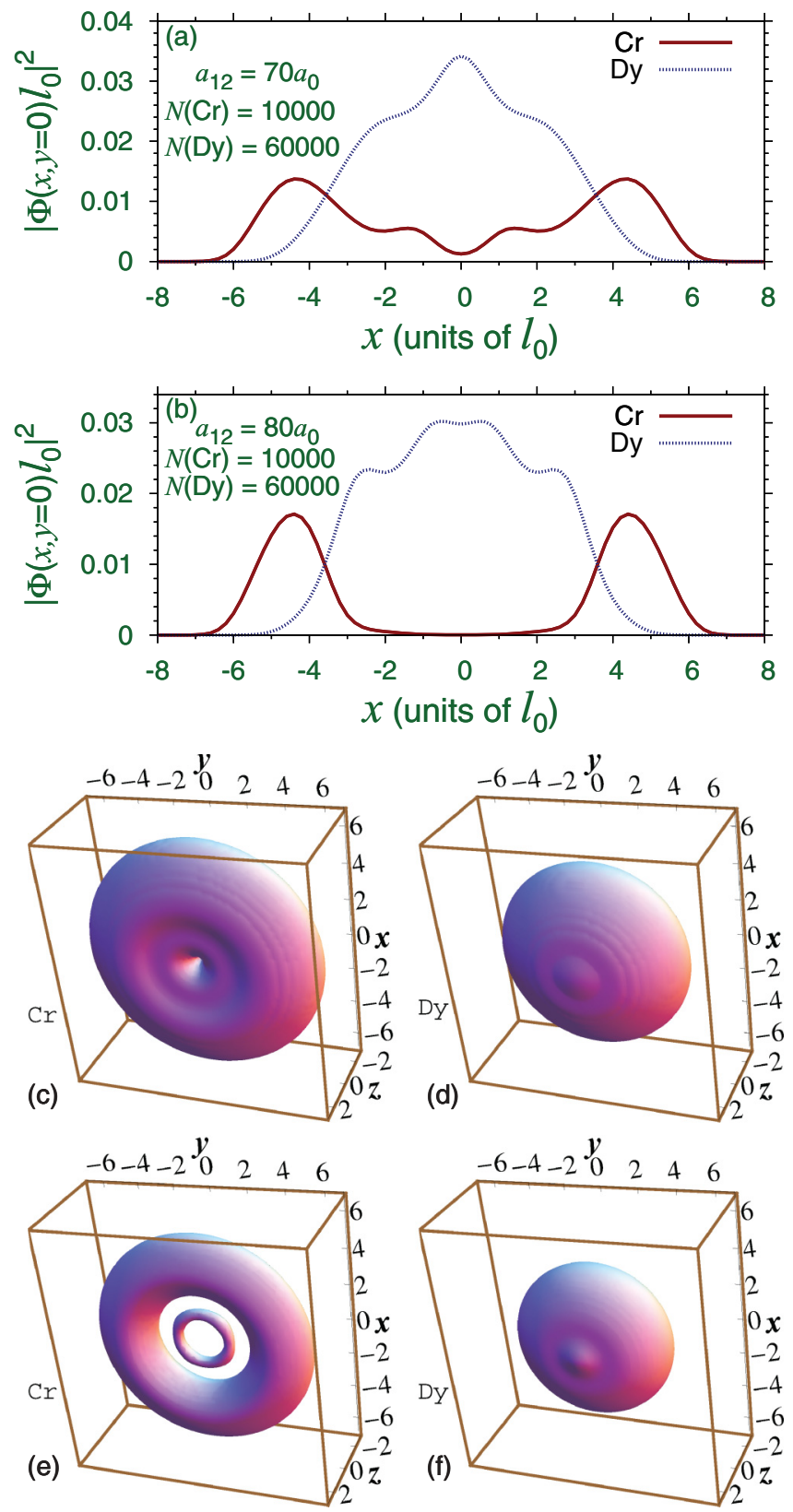

FIG. 7. (Color online) Two-dimensional radial density along the $x$ axis $|\Phi(x, y=0)|^{2} \equiv \int d z|\phi(x, y=0, z)|^{2}$ of the binary ${ }^{52} \mathrm{Cr}^{164} \mathrm{Dy}$ BEC, for (a) $a_{12}=70 a_{0}$, (b) $a_{12}=80 a_{0}$. Three-dimensional contour plot of the (c) ${ }^{52} \mathrm{Cr}$ and (d) ${ }^{164}$ Dy densities $\left|\phi_{i}(x, y, z)\right|^{2}$ of the binary BEC of Fig. 3(a) for $a_{12}=70 a_{0}$ with density on contour $=0.0005$. The same densities of (e) ${ }^{52} \mathrm{Cr}$ and (f) ${ }^{164} \mathrm{Dy}$ with density on contour $=0.0025$. In all cases $N(\mathrm{Cr})=10000, N(\mathrm{Dy})=60000$. The lengths $x, y, z$ are in units of $l_{0}=1 \mu \mathrm{m}$ and $3 \mathrm{D}$ density in units of $l_{0}^{-3}$.

with the density $0.0025 l_{0}^{-3}$ on the contour. This corresponds to the number densities on the contour of 25 and $150 \mu \mathrm{m}^{-3}$ in ${ }^{52} \mathrm{Cr}$ and ${ }^{164} \mathrm{Dy}$, respectively, for $l_{0}=1 \mu \mathrm{m}$. Figures $7(\mathrm{e})$ and 7(f) thus show the structure in the interior of the BECs illustrated in Figs. 7(c) and 7(d). Along the radial direction in the $x-y$ plane, we find in Figs. 7(e) and 7(f) that the maximum of density of one component is accompanied by the minimum of density of the other component due to interspecies contact repulsion and the density of one component may 

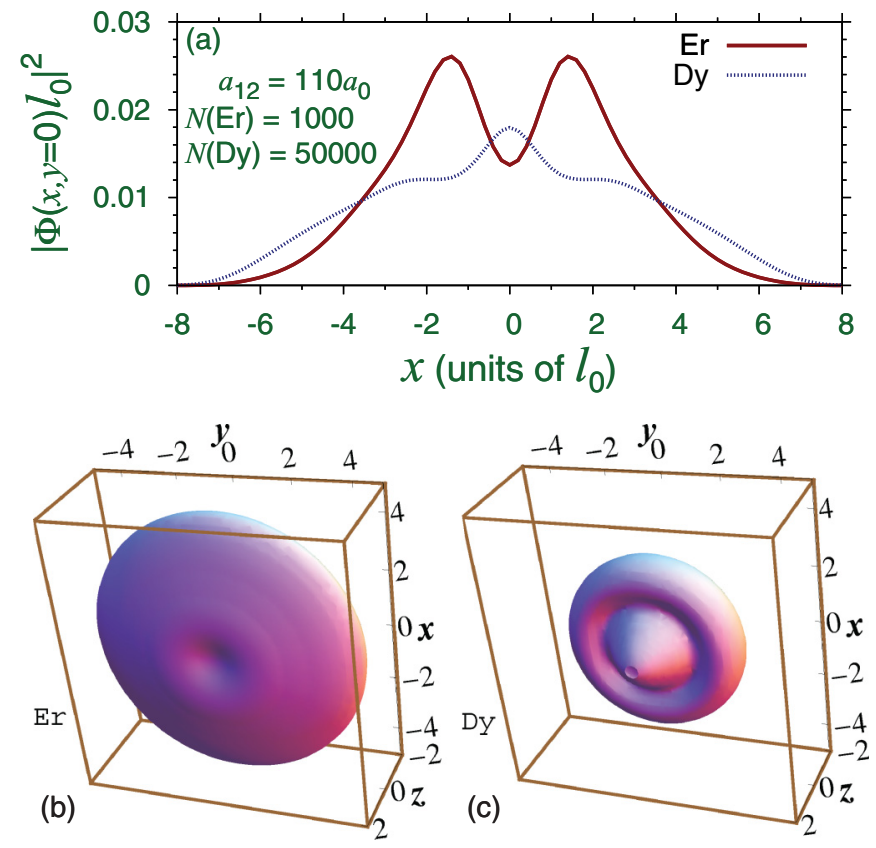

FIG. 8. (Color online) Two-dimensional radial density along the $x$ axis $|\Phi(x, y=0)|^{2} \equiv \int d z|\phi(x, y=0, z)|^{2}$ of the binary ${ }^{168} \mathrm{Er}-{ }^{164} \mathrm{Dy}$ $\mathrm{BEC}$ of $N(\mathrm{Er})=1000, N(\mathrm{Dy})=50000$, for (a) $a_{12}=110 a_{0}$. Three-dimensional contour plot of the (b) ${ }^{168} \mathrm{Er}$ and (c) ${ }^{164}$ Dy density $\left|\phi_{i}(x, y, z)\right|^{2}$ of the binary BEC of (a). Density on contour $=0.0025$. The lengths $x, y, z$ are in units of $l_{0}=0.5 \mu \mathrm{m}$ and 3D density in units of $l_{0}^{-3}$.

have several local maxima. The global maxima in densities of Figs. 7(e) and 7(f) are $0.0064 l_{0}^{-3}$ and $0.013 l_{0}^{-3}$, respectively, corresponding to atom densities of 64 and $780 \mu \mathrm{m}^{-3}$ for ${ }^{52} \mathrm{Cr}$ and ${ }^{164}$ Dy.

Finally, we study the distinct structure in densities of binary dipolar BECs with a smaller number ${ }^{168} \mathrm{Er}$ atoms in the shaded region of Fig. 2(b). In particular we consider the partially mixed configuration with $a_{12}=110 a_{0}, N(\mathrm{Er})=1000, N(\mathrm{Dy})$ $=50000$. In Fig. 8(a), we illustrate the $2 \mathrm{D}$ radial density along $x$ axis $|\Phi(x, y=0)|^{2} \equiv \int d z|\phi(x, y=0, z)|^{2}$ of this binary ${ }^{168}$ Er- ${ }^{164}$ Dy BEC. In Figs. 8(b) and 8(c) the contour plots of the corresponding 3D densities of ${ }^{168} \mathrm{Er}$ and ${ }^{164} \mathrm{Dy}$ are shown with a cutoff density $0.0025 l_{0}^{-3}$ on the contour. The maximum densities inside the ${ }^{168} \mathrm{Er}$ and ${ }^{164} \mathrm{Dy}$ BECs are $0.014 l_{0}^{-3}$ and
$0.0038 l_{0}^{-3}$, respectively. In this case a biconcave shape has appeared in the density profile of ${ }^{168} \mathrm{Er}$ BEC in Fig. 8(b). A Saturn-ring-like profile in the density of ${ }^{164} \mathrm{Dy}$ BEC is shown in Fig. 8(c). The central high-density region of ${ }^{164}$ Dy BEC has expelled the ${ }^{168} \mathrm{Er}$ BEC from the central region, thus creating a biconcave shape in density. If we compare Figs. 4(a) and 4(b) with Figs. 8(b) and 8(c), we find that the roles of ${ }^{164} \mathrm{Dy}$ and ${ }^{168}$ Er BECs have interchanged. In Fig. 4(a), the ${ }^{168}$ Er BEC has a Saturn-ring-like profile, whereas, in Fig. 8(c), the ${ }^{164} \mathrm{Dy}$ $\mathrm{BEC}$ has similar profile. These profiles are a consequence of dipolar interaction.

\section{SUMMARY}

Using a mean-field description we studied the static properties of binary disk-shaped dipolar BECs ${ }^{168} \mathrm{Er}-{ }^{164} \mathrm{Dy}$ and ${ }^{52} \mathrm{Cr}$-Dy to search for the effect of interspecies and intraspecies dipolar interactions employing realistic values of interspecies and intraspecies dipolar interactions as of intraspecies scattering lengths. The yet unknown interspecies scattering length is considered as a variable parameter. The binary system is found to be stable for a number of atoms below a critical value. The stability domain for the system is illustrated in convenient phase plots involving a number of atoms of the species and the interspecies scattering length $a_{12}$. For small values of $a_{12}$ a mixed state (with overlapping phase) of the two species emerges and this state transforms into a demixed state (with separated phase) for larger $a_{12}$. Near the stability line, in the region of transition from the mixed to a demixed configuration distinct structures in $3 \mathrm{D}$ densities may appear in the form of the Saturn-ring-like shape or red-blood-cell-like biconcave shape. Such structures are a direct manifestations of the dipolar interaction. A similar biconcave shape in the density of a single-component dipolar BEC was found and related to roton instability, which is presumably also responsible for these structures in the binary dipolar BECs. After this investigation was finished we came to know about another recent work on binary dipolar BEC in $2 \mathrm{D}$ [30]. Our results could be relevant for planning future experiments on binary dipolar BEC.

\section{ACKNOWLEDGMENTS}

We thank FAPESP and CNPq (Brazil) for partial support.
[1] T. Lahaye et al., Rep. Prog. Phys. 72, 126401 (2009).

[2] T. Lahaye et al., Nature (London) 448, 672 (2007); A. Griesmaier, J. Stuhler, T. Koch, M. Fattori, T. Pfau, and S. Giovanazzi, Phys. Rev. Lett. 97, 250402 (2006).

[3] J. Stuhler, A. Griesmaier, T. Koch, M. Fattori, T. Pfau, S. Giovanazzi, P. Pedri, and L. Santos, Phys. Rev. Lett. 95, 150406 (2005).

[4] K. Goral, K. Rzazewski, and T. Pfau, Phys. Rev. A 61, 051601 (2000).

[5] T. Koch et al., Nature Phys. 4, 218 (2008).

[6] M. Lu, S. H. Youn, and B. L. Lev, Phys. Rev. Lett. 104, 063001 (2010); J. J. McClelland and J. L. Hanssen, ibid. 96, 143005
(2006); S. H. Youn, M. W. Lu, U. Ray, and B. V. Lev, Phys. Rev. A 82, 043425 (2010).

[7] M. Lu, N. Q. Burdick, Seo Ho Youn, and B. L. Lev, Phys. Rev. Lett. 107, 190401 (2011).

[8] K. Aikawa, A. Frisch, M. Mark, S. Baier, A. Rietzler, R. Grimm, and F. Ferlaino, Phys. Rev. Lett. 108, 210401 (2012).

[9] J. Doyle, B. Friedrich, R. V. Krems, and F. Masnou-Seeuws, Eur. Phys. J. D 31, 149 (2004); J. Deiglmayr, A. Grochola, M. Repp, K. Mörtlbauer, C. Glück, J. Lange, O. Dulieu, R. Wester, and M. Weidemüller, Phys. Rev. Lett. 101, 133004 (2008); M. H. G. de Miranda et al., Nature Phys. 7, 502 (2011). 
[10] N. G. Parker, C. Ticknor, A. M. Martin, and D. H. J. O’Dell, Phys. Rev. A 79, 013617 (2009); R. M. Wilson, S. Ronen, and J. L. Bohn, ibid. 80, 023614 (2009); N. G. Parker and D. H. J. O’Dell, ibid. 78, 041601 (2008); L. Santos, G. V. Shlyapnikov, P. Zoller, and M. Lewenstein, Phys. Rev. Lett. 85, 1791 (2000); C. Ticknor, N. G. Parker, A. Melatos, S. L. Cornish, D. H. J. O'Dell, and A. M. Martin, Phys. Rev. A 78, 061607 (2008); R. M. W. van Bijnen, A. J. Dow, D. H. J. O’Dell, N. G. Parker, and A. M. Martin, ibid. 80, 033617 (2009); A. Junginger, J. Main, G. Wunner, and T. Bartsch, ibid. 86, 023632 (2012).

[11] L. Santos, G. V. Shlyapnikov, and M. Lewenstein, Phys. Rev. Lett. 90, 250403 (2003); R. M. Wilson, S. Ronen, J. L. Bohn, and H. Pu, ibid. 100, 245302 (2008); M. Asad-uz-Zaman and D. Blume, Phys. Rev. A 83, 033616 (2011).

[12] T. Lahaye, J. Metz, B. Fröhlich, T. Koch, M. Meister, A. Griesmaier, T. Pfau, H. Saito, Y. Kawaguchi, and M. Ueda, Phys. Rev. Lett. 101, 080401 (2008).

[13] I. Tikhonenkov, B. A. Malomed, and A. Vardi, Phys. Rev. Lett. 100, 090406 (2008); S. K. Adhikari and P. Muruganandam, J. Phys. B 45, 045301 (2012); L. E. Young-S., P. Muruganandam, and S. K. Adhikari, ibid. 44, 101001 (2011); P. Muruganandam and S. K. Adhikari, ibid. 44, 121001 (2011); P. Köberle, D. Zajec, G. Wunner, and B. A. Malomed, Phys. Rev. A 85, 023630 (2012).

[14] R. M. W. van Bijnen, D. H. J. O’Dell, N. G. Parker, and A. M. Martin, Phys. Rev. Lett. 98, 150401 (2007); R. Kishor Kumar and P. Muruganandam, J. Phys. B 45, 215301 (2012); M. Abad, M. Guilleumas, R. Mayol, M. Pi, and D. M. Jezek, Phys. Rev. A 79, 063622 (2009).

[15] P. Muruganandam and S. K. Adhikari, Phys. Lett. A 376, 480 (2012); C. Krumnow and A. Pelster, Phys. Rev. A 84, 021608 (2011); G. Bismut, B. Laburthe-Tolra, E. Maréchal, P. Pedri, O. Gorceix, and L. Vernac, Phys. Rev. Lett. 109, 155302 (2012).
[16] R. M. Wilson, S. Ronen, and J. L. Bohn, Phys. Rev. Lett. 104, 094501 (2010).

[17] B. Capogrosso-Sansone, C. Trefzger, M. Lewenstein, P. Zoller, and G. Pupillo, Phys. Rev. Lett. 104, 125301 (2010).

[18] K. Lakomy, R. Nath, and L. Santos, Phys. Rev. A 85, 033618 (2012); S. K. Adhikari and P. Muruganandam, Phys. Lett. A 376, 2200 (2012).

[19] L. E. Young-S., S. K. Adhikari, and P. Muruganandam, Phys. Rev. A 85, 033619 (2012).

[20] E. Timmermans, Phys. Rev. Lett. 81, 5718 (1998); A. A. Svidzinsky and S. T. Chui, Phys. Rev. A 68, 013612 (2003); G. Gligorić, A. Maluckov, M. Stepić, L. Hadzievski, and B. A. Malomed, ibid. 82, 033624 (2010); P. Kuopanportti, J. A. M. Huhtamäki, and M. Möttönen, ibid. 85, 043613 (2012); K.-T. Xi, J. Li, and D.-N. Shi, ibid. 84, 013619 (2011).

[21] I. Corro, R. G. Scott, and A. M. Martin, Phys. Rev. A 80, 033609 (2009).

[22] Y. Takeuchi and H. Mori, Phys. Rev. A 72, 063617 (2005); S. K. Adhikari and L. Salasnich, ibid. 75, 053603 (2007).

[23] S. K. Adhikari, Phys. Rev. A 73, 043619 (2006).

[24] H. Saito, Y. Kawaguchi, and M. Ueda, Phys. Rev. Lett. 102, 230403 (2009)

[25] S. Ronen, D. C. E. Bortolotti, and J. L. Bohn, Phys. Rev. Lett. 98, 030406 (2007).

[26] S. Yi and L. You, Phys. Rev. A 63, 053607 (2001).

[27] P. Muruganandam and S. K. Adhikari, Comput. Phys. Commun. 180, 1888 (2009); D. Vudragovic, I. Vidanovic, A. Balaz, P. Muruganandam, and S. K. Adhikari, ibid. 183, 2021 (2012).

[28] K. Goral and L. Santos, Phys. Rev. A 66, 023613 (2002).

[29] S. K. Adhikari, Phys. Rev. A 85, 053631 (2012); F. Malet, G. M. Kavoulakis, and S. M. Reimann, ibid. 84, 043626 (2011).

[30] R. M. Wilson, C. Ticknor, J. L. Bohn, and E. Timmermans, Phys. Rev. A 86, 033606 (2012). 